\title{
Robust Model Predictive Control with Almost Zero Online Computation
}

\author{
Yan Yan and Longge Zhang *(1) \\ Department of Mathematics and Physics, North China Electric Power University, Baoding 071003, China; \\ yan-yan@ncepu.edu.cn \\ * Correspondence: longge@ncepu.edu.cn; Tel.: +86-0312-752-5353
}

Citation: Yan, Y.; Zhang, L. Robust Model Predictive Control with Almost Zero Online Computation. Mathematics 2021, 9, 242. https:// doi.org/10.3390/math9030242

Received: 20 December 2020

Accepted: 22 January 2021

Published: 26 January 2021

Publisher's Note: MDPI stays neutral with regard to jurisdictional claims in published maps and institutional affiliations.

\begin{abstract}
This paper provides a strategy for the problem of robust model predictive control of constrained, discrete-time systems with state and output disturbances. Using the linear matrix inequality (LMI) method, the nested geometric proportion asymptotically stable ellipsoid (GPASE) strategy is designed off-line, and then the designed shrinking ellipsoids strategy assures the system converges on the equivalent with an exponential convergence velocity. The biggest advantage of this method is the online computation is almost reduced to zero, which makes it possible to apply the designed control scheme not only to plants with slowly varying parameters, but also to fast ones. Finally, a simulation example shows the validity of the proposed technique.
\end{abstract}

Keywords: robust model predictive control; shrinking; linear matrix inequality; robust control

\section{Introduction}

Model predictive control (MPC) [1-3], as it has the ability of dealing with multivariable constrained control problems, is widely used in the process industries. Since most models are only approximations of the actual processes, it is important to MPC to be robust, and this forms an important issue denoted as robust model predictive control (RMPC) [4]. Some researches [5] address the RMPC problem as a min-max optimization problem and it is formulated as linear matrix inequality (LMI) forms, but the heavy online computational burden restricts it to slow dynamic systems or small-scale processes.

In the last 20 years, reducing the high computation of RMPC has been a hot topic and most of the approaches have the common characteristics of moving most of the computational burden off-line. Examples of these strategies include explicit MPC scheme [6], efficient MPC $[7,8]$, polytopic approximations of exact controllable sets $[9,10]$ and nested ellipsoids [11-14]. Via multi-parametric programming explicit MPC [15-18] can compute a solution with explicit form to the finite horizon MPC problem off-line. By focusing on the nested ellipsoids scheme, the corresponding nested ellipsoids and state-feedback gains are computed off-line. Online, at each sampling time the smallest ellipsoid containing the current state is chosen and the selected state-feedback gain is implemented [11]. From the same philosophy this result is directly extended: the minimization of a nominal quadratic index replaces the worst-case optimization [12] and for linear parameter varying systems a new type of off-line model predictive control strategy is presented [19].

As we all know, an effective controller should be robust, have low online computation and minimal regulating time. Most of the aforementioned approaches can move much computational burden off-line, but few strategies have all three characteristics. In [20], we developed a shrinking ellipsoids strategy for RMPC based on the idea of geometric proportion asymptotically stable ellipsoid (GPASE), but the strategy did not consider the state and output disturbance. In this article, we extended our strategy to the system with state and output disturbance. The main contributions of this paper are as follows: the first is nested geometric proportion asymptotically stable ellipsoids are designed based on LMI; the second is a scheme of RMPC for the uncertain discrete-time with state and output 
disturbance is designed, and the most important is the designed controller assures the smallest regulating time of the closed system. It is more robust than the previous strategy as it can deal with the state and output disturbance explicitly. Moreover, as the state can be shrunk into the smaller ellipsoid, the online computation has been almost reduced to zero.

The structure of the article is organized as follows. In Section 2 the problem statement and early results are given. In Section 3, the control strategy is proposed based on the designed nested GPASEs. A simulation example is presented in next section and the conclusion is drawn in the end.

Notation: The notation used is fairly standard. $R^{n}$ is the $n$-dimensional space of real valued vectors. $\|x(k)\|=\left(x(k)^{T} \cdot x(k)\right)^{1 / 2}$ is the Euclidean norm for a vector $x(k)$. The matrix inequality $A>B$ and $A \geq B$ represent $A-B$ positive definiteness and positive semi-definiteness for square symmetric matrices $A$ and $B . x(k+i \mid k), i \in\{0,1,2, \ldots\}$ is the predicted value $x(k)$ at a future prediction time $k+i$ predicted at real time $k ; x(k)$ or $x(k \mid k)$ is the measured or actual value of variable $x$ at real time $k$; Co denoting the convex hull; $*$ denotes the corresponding transpose of the lower block part of symmetric matrices.

\section{Problem Statement}

Consider the following uncertain discrete-time system:

$$
\begin{aligned}
& x(k+1)=A(k) x(k)+B(k) u(k)+D(k) w(k) \\
& y(k)=C(k) x(k)+E(k) v(k)
\end{aligned}
$$

with the input constraints $\|u(k+i \mid k)\| \leq u_{\max }, \quad i>0$,

where $u(k) \in R^{n_{u}}, \quad x(k) \in R^{n_{x}}, \quad y(k) \in R^{n_{y}}, \quad w(k) \in R^{n_{w}}$ and $v(k) \in R^{n_{v}}$ are input, state, state disturbance and output measurement noise, respectively. $D(k)$ and $E(k)$ are shaping matrices, the disturbance satisfies $\|v(k)\| \leq 1,\|w(k)\| \leq 1$ and satisfies $w(k) \in \Omega_{w}, v(k) \in \Omega_{v}$. When $\|w(k)\|=\|v(k)\|=0$, it is corresponding to the undisturbance condition. Assume that

$$
\begin{gathered}
{[A(k), B(k), D(k)] \in \operatorname{Co}\left\{\left[A_{1}, B_{1}, D_{1}\right],\left[A_{2}, B_{2}, D_{2}\right], \ldots,\left[A_{L_{1}}, B_{L_{1}}, D_{L_{1}}\right]\right\}=\Omega_{1}} \\
{[C(k), E(k)] \in \operatorname{Co}\left\{\left[C_{1}, E_{1}\right],\left[C_{2}, E_{2}\right], \cdots\left[C_{L_{2}}, E_{L_{2}}\right]\right\}=\Omega_{2}}
\end{gathered}
$$

where Co denotes the convex hull. In other words, if $[A, B, D] \in \Omega_{1}$ then, for some nonnegative $\lambda_{1}, \lambda_{2}, \cdots, \lambda_{n}$ summing to one, we have

$$
[A, B, D]=\sum_{i=1}^{L_{1}} \lambda_{i}\left[A_{i}, B_{i}, D_{i}\right]
$$

Suppose the disturbance and the state satisfy the following equation:

$$
w(k)^{T} w(k) \leq x(k)^{T} x(k) .
$$

Remark 1. In practice, the disturbance ought to be less than the state, so (4) is generally satisfied.

Problem 1: We aimed at synthesizing an RMPC by solving the following min-max problem at each time $k$ :

$$
\begin{gathered}
\min _{u(k+i \mid k), i=0,1, \ldots, m}^{\max } \quad[A(k+i), B(k+i), D(k+i)] \in \Omega_{1}, w(k) \in \Omega_{w}, v(k) \in \Omega_{v} J_{\infty}(k) \\
\text { where } J_{\infty}(k)=\sum_{i=0}^{\infty}\left[x(k+i \mid k)^{T} Q_{1} x(k+i \mid k)+u(k+i \mid k)^{T} R u(k+i \mid k)\right] .
\end{gathered}
$$


Consider a quadratic function $V(x(k))=x(k)^{T} P x(k), P>0$. At every sampling time $k$, suppose the following inequality is satisfied:

$$
\begin{gathered}
V(x(k+i+1 \mid k))-V(x(k+i \mid k)) \\
\leq-\left[x(k+i \mid k)^{T} Q_{1} x(k+i \mid k)+u(k+i \mid k)^{T} R u(k+i \mid k)\right]
\end{gathered}
$$

As $V(x(\infty \mid k))=0$, summing (6) from $i=0$ to $i=\infty$, we get

$$
-V(x(k \mid k))<-J_{\infty}(k) .
$$

Thus $\max _{[A(k+i), B(k+i), D(k+i)] \in \Omega_{1}, \quad w(k) \in \Omega_{w}, v(k) \in \Omega_{v}} J_{\infty}(k) \leq V(x(k \mid k))$. Then the designed RMPC is redefined to synthesize a state-feedback control law $u(k+i \mid k)=F x(k+i \mid k)$ to minimize the upper bound $V(x(k \mid k))$.

Theorem 1. The state-feedback matrix $F$ in the control law $u(k+i \mid k)=F x(k+i \mid k)$ at the sampling time $k$ of Problem 1 is given by $F=Y Q^{-1}$, where $Q=\gamma P(k)^{-1}>0$ and $Y$ can be obtained from the following problem's solution:

$$
\min _{\gamma, Q, Y} \gamma
$$

subject to:

$$
\begin{aligned}
& {\left[\begin{array}{cc}
1 & x(k \mid k)^{T} \\
x(k \mid k) & Q
\end{array}\right] \geq 0,} \\
& {\left[\begin{array}{ccccc}
Q & Y^{T} R^{1 / 2} & Q Q_{1}^{1 / 2} & Q & \left(A_{j} Q+B_{j} Y\right)^{T} \\
R^{1 / 2} Y & \gamma I & 0 & 0 & 0 \\
Q_{1}^{1 / 2} Q & 0 & \gamma I & 0 & 0 \\
Q & 0 & 0 & \gamma \lambda^{-1} & 0 \\
A_{j} Q+B_{j} Y & 0 & 0 & 0 & Q-D_{j} \gamma \lambda^{-1} D_{j}^{T}
\end{array}\right] \geq 0,}
\end{aligned}
$$

$$
\left|\begin{array}{ll}
u_{\max }^{2} I & Y \\
Y^{T} & Q
\end{array}\right| \geq 0
$$

Proof. The proof of Equation (8) is the same as Theorem 1 and its proof is in the Appendix in paper [5], and the proof of Equation (10) is the same as Equation (32) in paper [5].

Substituting $u(k+i \mid k)=F x(k+i \mid k)$ and (1), inequality (6) becomes

$\left[\begin{array}{c}x(k+i \mid k) \\ w(k+i)\end{array}\right]^{T}\left[\begin{array}{cc}\left(A_{j}+B_{j} F\right)^{T} P\left(A_{j}+B_{j} F\right)-P+Q_{1}+F^{T} R F & \left(A_{j}+B F\right)^{T} P D \\ D^{T} P\left(A_{j}+B_{j} F\right) & D^{T} P D\end{array}\right]\left[\begin{array}{c}x(k+i \mid k) \\ w(k+i)\end{array}\right] \leq 0$

with

$$
w(k+i)^{T} I w(k+i) \leq x(k+i \mid k)^{T} I x(k+i \mid k) .
$$

Using the S-procedure, it is easy to see that (11) and (12) are satisfied if $\exists \lambda>0$ such that

$$
\left[\begin{array}{cc}
\left(A_{j}+B_{j} F\right)^{T} P\left(A_{j}+B_{j} F\right)-P+Q_{1}+F^{T} R F+\lambda I & \left(A_{j}+B_{j} F\right)^{T} P D \\
D^{T} P\left(A_{j}+B_{j} F\right) & D^{T} P D-\lambda I
\end{array}\right] \leq 0 .
$$

With the aid of the Sherman-Morrison-Woodbury formula

$$
\left(A_{j}-U D_{j}^{-1} V\right)^{-1}=A_{j}^{-1}+A_{j}^{-1} U\left(D_{j}-V A_{j}^{-1} U\right)^{-1} V A_{j}^{-1}
$$


we can get

$$
\left(Q-D_{j} \gamma \lambda^{-1} D_{j}^{T}\right)^{-1}=Q^{-1}+Q^{-1} D_{j}\left(\lambda \gamma^{-1} I-D_{j}^{T} Q^{-1} D_{j}\right)^{-1} D_{j}^{T} Q^{-1} .
$$

Pre-multiplying $\left[\begin{array}{cc}Q & 0 \\ 0 & I\end{array}\right]^{T}$, post-multiplying $\left[\begin{array}{cc}Q & 0 \\ 0 & I\end{array}\right]$, and using the Schur complement, Equation (13) is equivalent to

$$
\begin{gathered}
\left(A_{j} Q+B_{j} Y\right)^{T} P\left(A_{j} Q+B_{j} Y\right)-\gamma Q+Q Q_{1} Q+Y^{T} R Y+\lambda Q^{T} Q \\
-\left(A_{j} Q+B_{j} Y\right)^{T} P D_{j}\left(D_{j}^{T} P D_{j}-\lambda I\right)^{-1} D_{j}^{T} P\left(A_{j} Q+B_{j} Y\right) \leq 0
\end{gathered}
$$

With Equation (15) it is equivalent to

$$
Q-\left[\begin{array}{llll}
Y^{T} R^{1 / 2} & Q Q_{1}^{1 / 2} & Q & \left(A_{j} Q+B_{j} Y\right)^{T}
\end{array}\right]\left[\begin{array}{ccccc}
\gamma^{-1} I & 0 & 0 & 0 \\
0 & \gamma^{-1} I & 0 & 0 \\
0 & 0 & \gamma^{-1} \lambda & 0 \\
0 & 0 & 0 & \left(Q-D_{j} \gamma \lambda^{-1} D_{j}^{T}\right)^{-1}
\end{array}\right]\left[\begin{array}{c}
R^{1 / 2} Y \\
Q_{1}^{1 / 2} Q \\
Q \\
\left(A_{j} Q+B_{j} Y\right)
\end{array}\right] \geq 0
$$

Using the Schur complement again we can get

$$
\left[\begin{array}{ccccc}
Q & Y^{T} R^{1 / 2} & Q Q_{1}^{1 / 2} & Q & \left(A_{j} Q+B_{j} Y\right)^{T} \\
R^{1 / 2} Y & \gamma I & 0 & 0 & 0 \\
Q_{1}^{1 / 2} Q & 0 & \gamma I & 0 & 0 \\
Q & 0 & 0 & \gamma \lambda^{-1} & 0 \\
A_{j} Q+B_{j} Y & 0 & 0 & 0 & Q-D_{j} \gamma \lambda^{-1} D_{j}^{T}
\end{array}\right] \geq 0 .
$$

$j=1,2 \cdots, L_{1}$, which is affine in $\left[\begin{array}{lll}A & B & D\end{array}\right]$, so Equation (6) is equivalent to (9), and completes the proof.

Thus, the problem can be solved by

$$
\min _{\gamma, Q, X, Y, Z} \gamma \text {, s.t. (8), (9), (10). }
$$

Obviously, it is very important to reduce the online computation as the aforementioned method has prohibitive computation. One important method is the off-line robust MPC strategy [11,12]. We proposed a shrinking method of RMPC [20], but the method only considered the system without state and output disturbance. In practice, most of the dynamic systems are influence by the state and output disturbance/noise. If the disturbance is not considered, the control performance will be very poor, even the system is not stable, so we propose the shrinking ellipsoids strategy for robust model predictive control with state and output disturbance.

\section{The Synthesis of the Shrinking Ellipsoids Strategy}

In this section, we discuss the shrinking ellipsoids strategy for robust MPC with state and output disturbance. First, the GPASE is designed off-line. Then the synthesis of shrinking ellipsoids strategy for linear parameter-varying (LPV) system is proposed.

\subsection{Design of GPASE}

Definition 1. [20]. For discrete dynamical system $x(k+1)=f(x(k))$, the ellipsoidE $=$ $\left\{x(k) \in R^{n} \mid x(k)^{T} Q^{-1} x(k) \leq 1\right\}$ is a geometric proportion asymptotically stable ellipsoid, if whenever $x(k) \in E$, then $x(k+1) \in E_{\rho}$, where $E_{\rho}=\left\{x(k) \in R^{n} \mid x(k)^{T} Q^{-1} x(k) \leq \rho^{2}\right\}$, 
$0<\rho<1$. It is known that the rate of the convergence can be influenced by setting a minimum convergence rate on the state $\|x(k)\| \leq \rho^{k}|| x(0) \| \quad(0<\rho<1)$ as follows

$$
x(k+i+1 \mid k)^{T} Q^{-1} x(k+i+1 \mid k) \leq \rho^{2} x(k+i \mid k)^{T} Q^{-1} x(k+i \mid k)
$$

for any $[A(k), B(k), D(k)] \in \Omega$.

Lemma 1. For system (1), if it has a minimum convergence rate of (20), the following LMI must be satisfied:

$$
\left[\begin{array}{cc}
\rho^{2} Q & * \\
A_{i} Q+B_{i} Y & Q
\end{array}\right] \geq 0, \quad i=1, \cdots, L_{1} .
$$

Proof. as $x(k+i+1 \mid k)^{T} Q^{-1} x(k+i+1 \mid k) \leq \rho^{2} x(k+i \mid k)^{T} Q^{-1} x(k+i \mid k)$

We can get

$$
\begin{gathered}
{[(A+B F) x(k+i \mid k)+D(k+i) w(k+i)]^{T} Q^{-1}[(A+B F) x(k+i \mid k)+D(k+i) w(k+i)]} \\
\leq \rho^{2} x(k+i \mid k)^{T} Q^{-1} x(k+i \mid k)
\end{gathered}
$$

Then

$$
\begin{gathered}
x(k+i \mid k)^{T}(A+B F) Q^{-1}(A+B F) x(k+i \mid k)+x(k+i \mid k)^{T}(A+B F)^{T} Q^{-1} D w(k+i) \\
w(k+i)^{T} D^{T} Q^{-1} D(A+B F) x(k+i \mid k)+w(k+i)^{T} D^{T} Q^{-1} D w(k+i) \\
\leq \rho^{2} x(k+i \mid k)^{T} Q^{-1} x(k+i \mid k)
\end{gathered}
$$

That is

$$
\begin{aligned}
{[x(k+i \mid k)} & w(k+i)]^{T}\left[\begin{array}{cc}
(A+B F)^{T} Q^{-1}(A+B F)-\rho^{2} Q^{-1} & (A+B F)^{T} P D \\
D^{T} P(A+B F) & D^{T} Q^{-1} D
\end{array}\right]\left[\begin{array}{c}
x(k+i \mid k) \\
w(k+i)
\end{array}\right] \leq 0 . \\
& \text { It is equilibrant to }\left[\begin{array}{cc}
(A+B F)^{T} Q^{-1}(A+B F)-\rho^{2} Q^{-1} & (A+B F)^{T} P D \\
D^{T} P(A+B F) & D^{T} Q^{-1} D
\end{array}\right] \leq 0 .
\end{aligned}
$$

Pre-multiplying $\left[\begin{array}{cc}Q & 0 \\ 0 & I\end{array}\right]^{T}$, post-multiplying $\left[\begin{array}{cc}Q & 0 \\ 0 & I\end{array}\right]$, and using the Schur complement $(A Q+B Y)^{T} Q^{-1}(A Q+B Y)-\rho^{2} Q-(A Q+B Y)^{T} P D\left(D^{T} Q^{-1} D\right)^{-1} D^{T} P(A+B Y) \leq 0$.

That is $\rho^{2} Q-(A Q+B Y)^{T} Q^{-1}(A Q+B Y)+(A Q+B Y)^{T} P D\left(D^{T} Q^{-1} D\right)^{-1} D^{T} P(A+B Y) \geq 0$.

As $Q$ is positive, so $(A Q+B Y)^{T} P D\left(D^{T} Q^{-1} D\right)^{-1} D^{T} P(A+B Y) \geq 0$. So it only needs $\rho^{2} Q-(A Q+B Y)^{T} Q^{-1}(A Q+B Y) \geq 0$.

Then using the Schur complement again we can get

$$
\left[\begin{array}{cc}
\rho^{2} Q & * \\
A_{i} Q+B_{i} Y & Q
\end{array}\right] \geq 0, \quad i=1, \cdots, L_{1} .
$$

Assumption 1. Assume the uncertain system (1) with LMIs has the proper feasible initial region. We assume the original point is the equilibrium point, so it ought to be in the feasible initial region. If the original point is not the equilibrium point, we can make a transformation $(X(k)=x(k)-\bar{x}(k)$, $\bar{x}(k)$ is the original system's equilibrium point, and $X(k)$ is the transformed system), so that the origin is the equilibrium point of the new system. 


\begin{abstract}
Algorithm 1. (GPASE off-line design). Consider the uncertain system (1), a sequence of minimizers $\gamma_{i}, Q_{i}, X_{i}, Y_{i}$ and $Z_{i}$ are generated based on the proper feasible initial state $x_{1}$ off-line
\end{abstract} as follows:

Set $i:=1$.

Step 1 : Compute $\gamma_{i}, Q_{i}, X_{i}, Y_{i}, Z_{i}$ at $x_{i}(k)$

by (19), with additional LMIs $(21)$ and $\rho^{2} Q_{i}<Q_{i+1}<Q_{i}$; store $F_{i}$.

Step 2: If $i<N$, choose a state $x_{i+1}(k)$ satisfied $x_{i+1}^{T}(k) Q_{i}^{-1} x_{i+1}(k)=\rho^{2} x_{i}^{T}(k) Q_{i}^{-1} x_{i}(k)$ . Let $i:=i+1$, go to step 1 .

Remark 2. $N$ is the number of designed off-line ellipsoids. The bigger $N$ needs more off-line computation and more storage, and it depends on the designed shrinking ratio $\rho$.

Lemma 2. [20]. Suppose the positive definite matrices satisfy $0<\rho^{2} Q_{i}<Q_{i+1}<Q_{i}$ $(0<\rho<1)$, then $E_{i, \rho} \subset E_{i+1} \subset E_{i}$, where $E_{i}=\left\{x(k) \in R^{n} \mid x^{T}(k) Q_{i}^{-1} x(k) \leq 1\right\}$ and $E_{i, \rho}=\left\{x(k) \in R^{n} \mid x^{T}(k) Q_{i}^{-1} x(k) \leq \rho^{2}\right\}$.

The relation of $E_{i, \rho}, E_{i+1}$ and $E_{i}$ is shown in Figure 1.

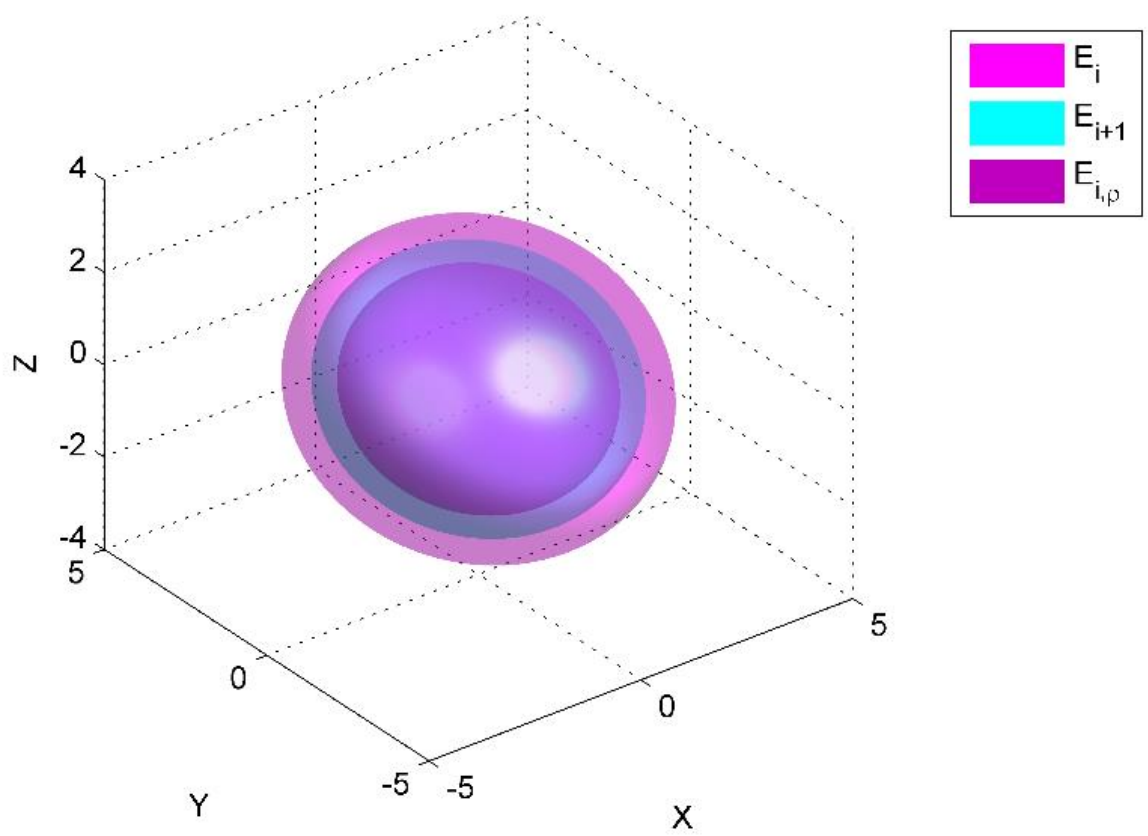

Figure 1. Relation of $E_{i, \rho}, E_{i+1}$ and $E_{i}: E_{i, \rho} \subset E_{i+1} \subset E_{1}$.

Remark 3. For the convenience of operation, the corresponding points can be selected on the $x$-axis. That is to say, all the coordinates of $x_{i}$ are zero except the first one. For finding the largest feasible region, the method of bisection will be used, that is to say, we will find $x_{1}$ on the $x$-axis using the method of bisection.

Remark 4. The number $N$ has an influence on the feasibility and the off-line computational load. If $N$ is too small, Algorithm 1 will have no feasible solution; if $N$ is too large, it will increase the off-line computational load.

\title{
3.2. Shrinking Ellipsoids Strategy
}

Based on the GPASE designed in 1, the shrinking ellipsoids strategy for the LPV system is proposed as follows. 


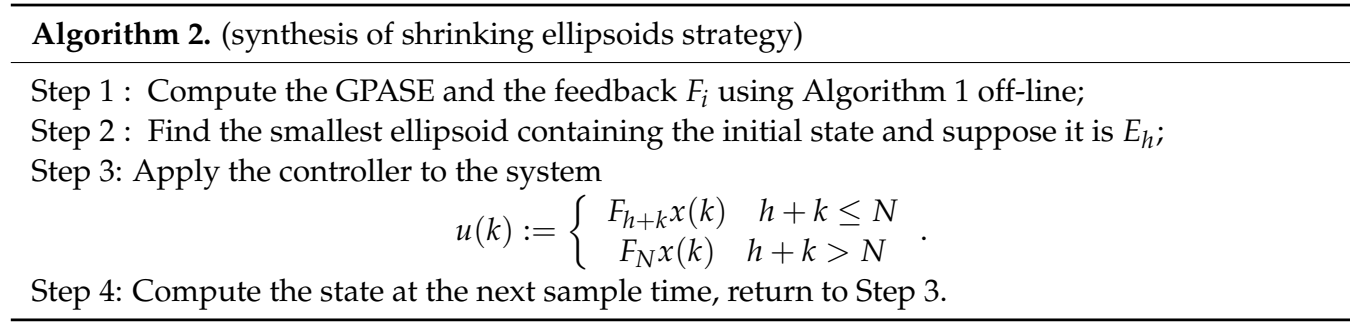

Theorem 2. For dynamical system (1), the shrinking ellipsoids strategy Algorithm 2 asymptotically stabilizes the closed-loop system.

Proof. With regard to the off-line minimization at $x_{i}(k), i=1,2, \cdots, N$. It is ensured that its convergence speed rate is not less than $\rho$ by the additional LMI (21). So, if $x(k) \in E_{i}=$ $\left\{x(k) \in R^{n} \mid x^{T}(k) Q_{i}^{-1} x(k) \leq 1\right\}$, then $x(k+1) \in E_{i, \rho}=\left\{x(k) \in R^{n} \mid x^{T}(k) Q_{i}^{-1} x(k) \leq \rho^{2}\right\}$. From Lemma 2, the designed controller can assure the state to be shrunk into the inner ellipsoid, that is, the control law for the ellipsoid $E_{i}$ is guaranteed to assure the state within $E_{i}$ and converge into the ellipsoid $E_{i+1}$, and so on. Lastly, $u_{N}=F_{N} x(k)$ is guaranteed to be in $E_{N}$ and converge to the equivalent point and the asymptotic stability of the closed-loop system is realized in the end.

Remark 5. Regarding the GPASE, the online computation only finds the smallest ellipsoid containing the initial state. Once it is found, the state is shrunk into the smaller ellipsoid, so the online computation is almost zero. So the number of the preset points only affects the off-line computation but not the online calculation.

Remark 6. The polyhedral invariant set can easily deal with non-symmetric constraints, and in this paper the designed algorithm is based on the ellipsoidal sets. So Algorithm 2 is usually more conservative than a polyhedral invariant set, so the strategy is more conservative than the method in [21].

\section{Simulation Example}

Example. Consider the followed system

$$
\begin{gathered}
A(k)=\left[\begin{array}{cc}
0.385 & 0.33 \\
0.21+\alpha(k) & 0.59
\end{array}\right], B(k)=\left[\begin{array}{c}
1+\alpha(k) \\
0
\end{array}\right] \\
C(k)=\left[\begin{array}{ll}
0.3 & 0.3
\end{array}\right], D(k)=0.2 \\
E(k)=0.3, \quad w(k)=\sin (0.3 x(k)), v(k)=\cos (0.5 x(k))
\end{gathered}
$$

where $\alpha(k)$ is an uncertain parameter satisfying $|\alpha(k)| \leq 0.11$. In this case, $A_{1}=\left[\begin{array}{cc}0.385 & 0.33 \\ 0.32 & 0.59\end{array}\right], B_{1}=\left[\begin{array}{c}1.11 \\ 0\end{array}\right], A_{2}=\left[\begin{array}{cc}0.385 & 0.33 \\ 0.10 & 0.59\end{array}\right], B_{2}=\left[\begin{array}{c}0.89 \\ 0\end{array}\right], D_{1}=D_{2}=$ $D=0.2$. The polytopic uncertain set is given as follows

$$
\Omega=\operatorname{Co}\left\{\left[A_{1}, B_{1}, D\right],\left[A_{1}, B_{2}, D\right],\left[A_{2}, B_{1}, D\right],\left[A_{2}, B_{2}, D\right]\right\} .
$$

First, the online RMPC is used and the initial condition is chosen as $x_{0}=[3 ; 2]$. When $k=30$, the system can be controlled to the equilibrium point. When the system is simulated on the MacBook (Retina, 12-inch, 2017) with the CPU $1.2 \mathrm{GHz}$ Intel Core m3, the elapsed time is $3.189613 \mathrm{~s}$. The system's states and the random variable are shown as Figures 2 and 3, respectively. 

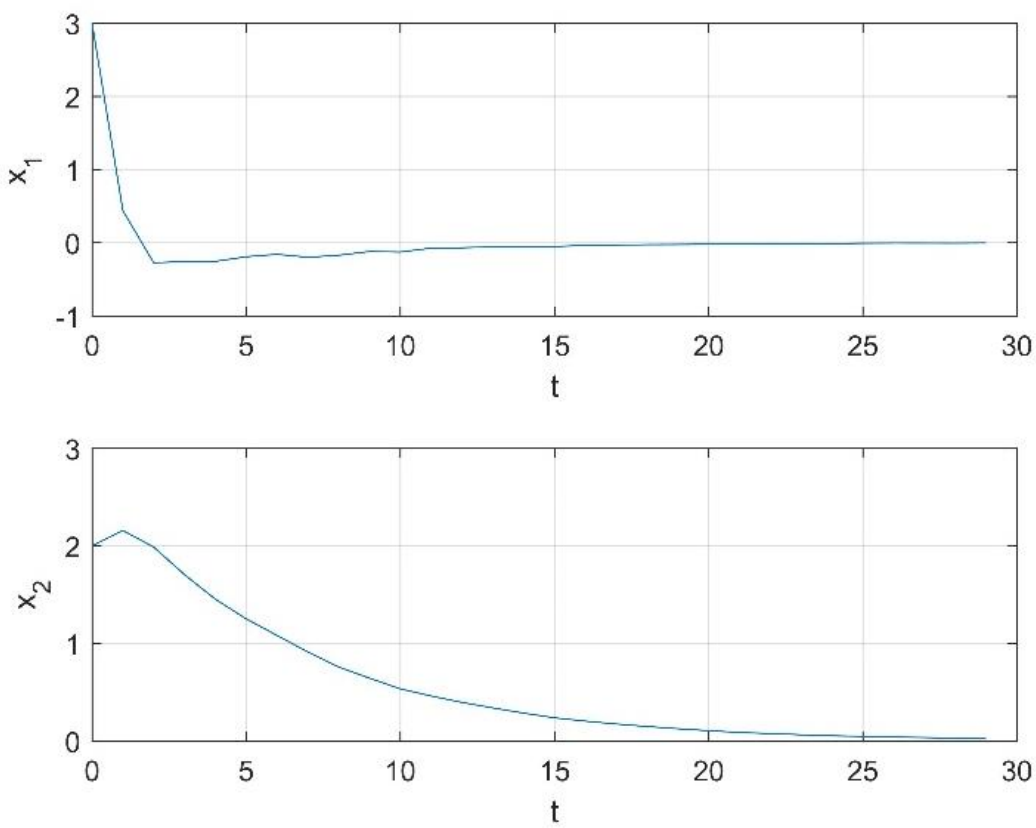

Figure 2. The states under the online controller.

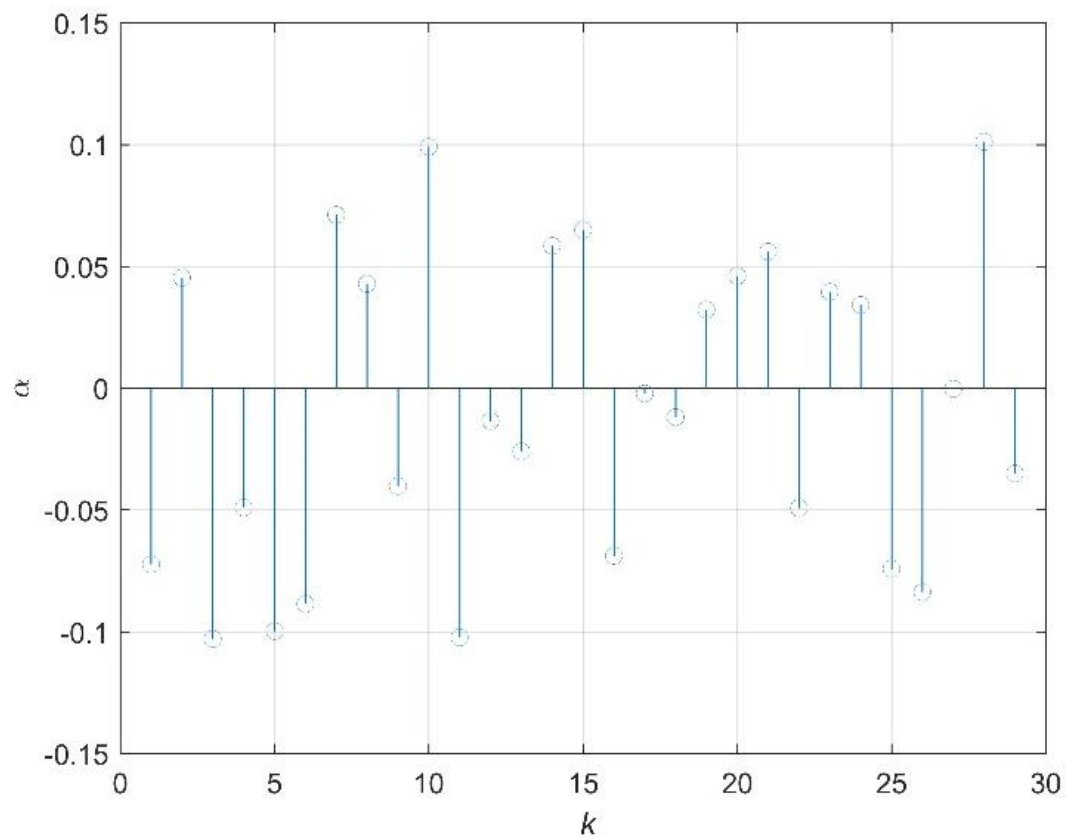

Figure 3. The random variable of $\alpha(k)$.

If the Algorithm 2 is applied to the system with the same random variable the system's states are shown as Figure 4. The nested ellipsoids are shown as Figure 5. The online computing time is almost zero as most of the computation time is moved off-line, and the next step is only applying the pre-computed controller on the system. From Figures 2 and 4 we know that the performance of the designed off-line strategy is almost the same with the online controller, but the computation burden is reduced dramatically. As far as the authors know, most of the RMPC method of the system with state and output disturbance is output RMPC, so in this condition we cannot compare this method with other algorithms with the same simulation. 

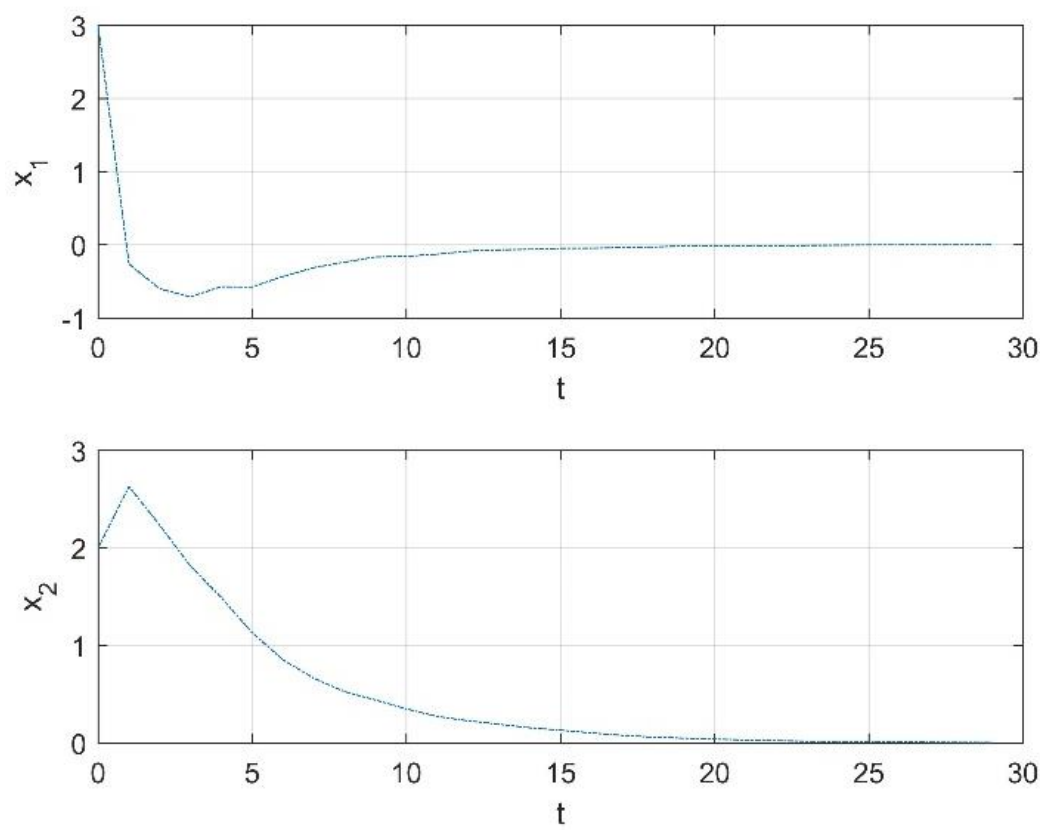

Figure 4. The states under controller of Algorithm 2.

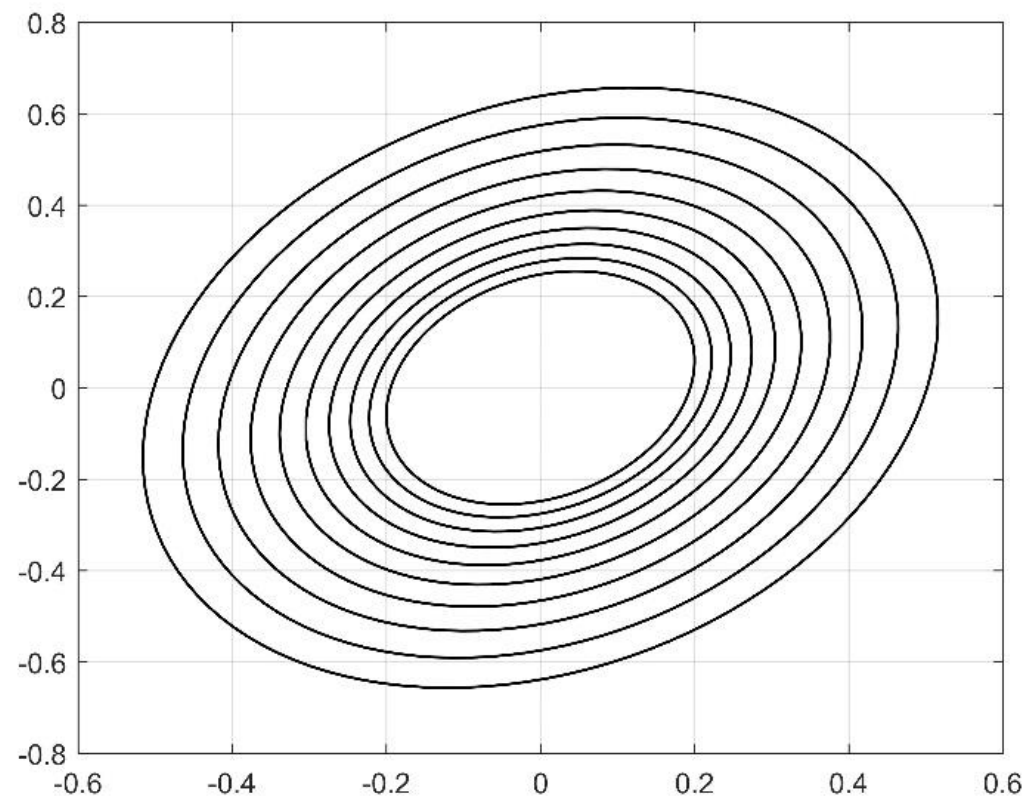

Figure 5. The designed nested ellipsoids.

\section{Conclusions}

This paper presents an off-line strategy for robust constrained MPC with state and output disturbances. The concept of nested geometric proportion asymptotically stable ellipsoids is first proposed and designed through adding an additional LMI to the traditional RMPC problem. The GPASE strategy is designed off-line and can assure the system converges to the equivalent. With this method the online computation is almost reduced to zero, which makes it possible to apply it to the broad areas. The strategy in this article can deal with the system with state and output disturbances, and these disturbances often exist on real systems, so the online calculation is reduced dramatically. The usefulness and effectiveness of the method is illustrated with a simulation example. 
Author Contributions: Writing-original draft, Y.Y.; Software, L.Z. All authors have read and agreed to the published version of the manuscript.

Funding: This work is supported by the fund of the National Nature Science Foundation of China Scholarship Council under grant 201706735054, the Hebei Provincial Natural Science Foundation under Grant F2016502025.

Institutional Review Board Statement: Not applicable.

Informed Consent Statement: Not applicable.

Data Availability Statement: Data sharing not applicable.

Conflicts of Interest: The authors declare no conflict of interest.

\section{References}

1. Grüne, L.; Pannek, J. Nonlinear model predictive control. In Nonlinear Model Predictive Control; Springer: Berlin/Heidelberg, Germany, 2017; pp. 45-69.

2. Mayne, D.Q.; Rawlings, J.B.; Rao, C.V.; Scokaert, P.O.J.A. Constrained model predictive control: Stability and optimality. Automatica 2000, 36, 789-814. [CrossRef]

3. Lorenzen, M.; Cannon, M.; Allgöwer, F.J.A. Robust MPC with recursive model update. Automatica 2019, 103, 461-471. [CrossRef]

4. Eliasi, H.; Rahmati, S. Robust model predictive control for a class of discrete nonlinear systems. J. Nonlinear Syst. Electric. Eng. 2020, 6, 104-118.

5. Kothare, M.V.; Balakrishnan, V.; Morari, M.J.A. Robust constrained model predictive control using linear matrix inequalities. Automatica 1996, 32, 1361-1379. [CrossRef]

6. Lee, J.; Chang, H.-J.; Ahn, H.-S. Explicit MPC for column-type EPS systems. IET Electr. Power Appl. 2019, 14, 91-100. [CrossRef]

7. Kouvaritakis, B.; Rossiter, J.; Schuurmans, J. Efficient robust predictive control. IEEE Trans. Automat. Contr. 2000, 45, 1545-1549. [CrossRef]

8. Abdelbaky, M.A.; Liu, X.; Jiang, D.J.R.E. Design and implementation of partial offline fuzzy model-predictive pitch controller for large-scale wind-turbines. Renew. Energy 2020, 145, 981-996. [CrossRef]

9. Diehl, M.; Bjornberg, J. Robust dynamic programming for min-max model predictive control of constrained uncertain systems. IEEE Trans. Automat. Contr. 2004, 49, 2253-2257. [CrossRef]

10. Ma, A.; Cheng, Q.; Liu, K.; Xia, Y. Time optimal model predictive control for linear systems based on ellipsoidal and polytopic sets. Renew. Energy 2019, 1-9. [CrossRef]

11. Wan, Z.; Kothare, M.V. An efficient off-line formulation of robust model predictive control using linear matrix inequalities. Automatica 2003, 39, 837-846. [CrossRef]

12. Ding, B.; Xi, Y.; Cychowski, M.T.; O’Mahony, T. Improving off-line approach to robust MPC based-on nominal performance cost. Automatica 2007, 43, 158-163. [CrossRef]

13. Zhang, L.G.; Liu, X.J.; Kong, X.B. State estimators for uncertain linear systems with different disturbance/Noise using quadratic boundedness. J. Appl. Math. 2012, 1-11. Available online: https://www.hindawi.com/journals/jam/2012/101353 (accessed on 3 June 2012). [CrossRef]

14. Longge, Z.; Yan, Y. Robust shrinking ellipsoid model predictive control for linear parameter varying system. PLoS ONE 2017, 12, e0178625. [CrossRef] [PubMed]

15. Avraamidou, S.; Pistikopoulos, E.N. A multi-parametric bi-level optimization strategy for hierarchical model predictive control. In Computer Aided Chemical Engineering; Elsevier: Amsterdam, The Netherlands, 2017; pp. 1591-1596.

16. Diangelakis, N.A.; Oberdieck, R.; Pistikopoulos, E.N. Explicit (Offline) optimization for MPC. In Handbook of Model Predictive Control; Springer: Berlin/Heidelberg, Germany, 2019; pp. 359-385.

17. Taghavipour, A.; Vajedi, M.; Azad, N.L. Multi-parametric predictive control. In Intelligent Control of Connected Plug-in Hybrid Electric Vehicles; Springer: Berlin/Heidelberg, Germany, 2019; pp. 79-102.

18. Charitopoulos, V.M.; Papageorgiou, L.G.; Dua, V.J. Closed-loop integration of planning, scheduling and multi-parametric nonlinear control. Comput Chem Eng. 2019, 122, 172-192. [CrossRef]

19. Bumroongsri, P.; Kheawhom, S. An ellipsoidal off-line model predictive control strategy for linear parameter varying systems with applications in chemical processes. Syst. Control. Lett. 2012, 61, 435-442. [CrossRef]

20. Zhang, L.G. Automatic offline formulation of robust model predictive control based on linear matrix inequalities method. Abstr. Appl. Anal. 2013, 1-9. [CrossRef]

21. Shokrollahi, A.; Shamaghdari, S. Offline robust model prsedictive control for lipschitz non-linear systems using polyhedral invariant sets. Asian J. Control 2020, 22, 288-296. [CrossRef] 